\title{
Multi-Track Programming Competitions with Scratch
}

\author{
Jason Arnold Heather Bort Ryan Naugle Casey O'Hare Dennis Brylow \\ Department of Mathematics, Statistics and Computer Science \\ Marquette University \\ 1313 West Wisconsin Avenue \\ Milwaukee, WI 53201-1881 \\ firstname.lastname@marquette.edu
}

\begin{abstract}
High school programming competitions often follow the traditional model of collegiate competitions, exemplified by the ACM International Collegiate Programming Contest (ICPC) This tradition has been reinforced by the nature of Advanced Placement Computer Science (AP CS A), for which ICPCstyle problems are considered an excellent practice regimen. As more and more students in high school computer science courses approach the field from broader starting points, such as Exploring Computer Science (ECS), or the new AP CS Principles course, an analogous structure for high school outreach events becomes of greater importance.

This paper describes our work on developing a Scratchbased alternative competition for high school students, that can be run in parallel with a traditional morning of ICPCstyle problems.
\end{abstract}

\section{Keywords}

Scratch; programming competition; K-12 outreach

\section{INTRODUCTION}

Computer programming competitions for high school students are a time-tested method of outreach and engagement that have been widely used by academia and industry.

Universities organize and execute programming competitions for high school students for several reasons:

1. Competitions raise the visibility of a campus and its academic computing offerings among future students,

2. Competitions offer an opportunity for universities to directly encourage promising future students,

3. Bringing teams to campus offers a rare window of opportunity for networking and professional development for those teams' computer science teachers, and

4. Many college students view helping run such a competition as worthy community service, and/or as preparation for the collegiate-level competition.

Conversely, high school computer science students and their teachers attend programming competitions because:

Permission to make digital or hard copies of all or part of this work for personal or classroom use is granted without fee provided that copies are not made or distributed for profit or commercial advantage and that copies bear this notice and the full citation on the first page. Copyrights for components of this work owned by others than ACM must be honored. Abstracting with credit is permitted. To copy otherwise, or republish, to post on servers or to redistribute to lists, requires prior specific permission and/or a fee. Request permissions from permissions@ acm.org.

SIGCSE '16, March 02-05, 2016, Memphis, TN, USA

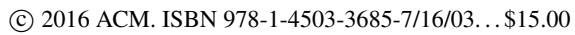

DOI: http://dx.doi.org/10.1145/2839509.2844634
1. Students who take up an interest in computer science often would like a place to hone their skills and be recognized for their accomplishments,

2. Students are eager to see college campuses and meet college students in a likely area of future study, and

3. Students often view such competitions as valuable practice for subsequent, high-stakes testing such as the Advanced Placement exam in Computer Science.

In most states in the U.S., more than $85 \%$ of high schools have no computer science teacher, and thus no computer science courses. High school programming contests held at college campuses in our region have been attractive only to a very narrow, elite band of high schools. Those schools are overwhelmingly suburban schools in orbit about metropolitan areas. Participants from those schools tend to be mostly white or Asian males, from middle-class backgrounds. Many are planning to major in computer science already.

Beginning in 2014, a National Science Foundation grant project started to roll out professional development for area teachers in schools with no computer science presence. Due to this project, 18 new school districts in our state began teaching ECS [6], and over 600 new high school students were taking computer science for the first time. We quickly realized that our traditional model of high school programming competitions was a poor match for the wave of new students entering computer science with broader introductory courses.

In response, we have adapted our traditionally-structured high school programming contest to make it more attractive to this new population of students. We added a second, parallel track to the traditional Java-based competition that uses the Scratch programming language to welcome students from courses such as ECS, or the new AP CS Principles [4] (AP CSP) course. Broadening the reach of this outreach beyond those schools with existing, well-established programs that culminate in Advanced Placement CS A is essential to engaging a more diverse set of participants. Our novel structure requires problems that can be quickly judged, but allow open-ended solutions rewarding creativity and artistic merit. Most significantly, two of the 11 schools participating this year were coached by brand-new ECS teachers, from districts that previously had no CS teacher or courses.

Although years of experience with traditional contest models have produced robust contest management software ${ }^{1}$, a rich pool of existing and reusable contest tasks ${ }^{2}$, and deep institutional knowledge about how to successfully engineer

\footnotetext{
$\overline{{ }^{1} \text { http://www.ecs.csus.edu/pc2/ }}$

${ }^{2}$ http://uva.onlinejudge.org/
} 
and execute an event, there has been little published work on designing a Scratch-based competition with short-term, open-ended challenge tasks that must run in parallel with a traditional ICPC-style Java track.

This paper presents the first two years of our experience with the challenge of designing and fielding just such a competition.

\section{BACKGROUND}

Programming competitions have been used to foster and encourage student interest in computing since at least the early 1970s [2]. In this section, we briefly summarize the large body of prior and related work.

\subsection{Collegiate Programming Competitions}

The Association for Computing Machinery (ACM) International Collegiate Programming Competition (ICPC) is among the oldest and most respected institutions for bringing computing students together in competition from around the world. The typical format has teams of three students working on eight or more complex algorithmic thinking tasks with a five-hour time limit and only one computer. [2]

Regional and local competitions at the collegiate level frequently follow the model of the ICPC, using the vast repository of past contest tasks as both practice and competition problems.

The International Olympiad in Informatics [11] is billed as, "the most prestigious international algorithmic programming competition at the high-school level". National bodies, such as the USA Computing Olympiad [18], hold internal competitions to select a team of delegates to participate in the international competition, not unlike the Olympic Games for athletics.

For USACO, the internal rounds consist of online competitions, supplemented by many hours of free, online training.

Separately, the American Computer Science League [1] offers asynchronous, school-based competition with both a written exam component and a solo programming component. The asynchrony allows ACSL to scale to many schools, by allowing individual teachers to offer the competition during the school day at a time that suits the local context in the high school building.

A wide variety of corporate-sponsored coding contests and web portals compete for the attention of high school students with a strong interest in computer science and related fields. A thorough survey of the landscape of programming competitions is provided in [8].

With many decades of experiences running programming competitions for high school students, more recent research has sought to classify and clarify the categories of competitions and tasks commonly found. Pohl [16] provides a taxonomy of computer science competitions, contests and challenges. Ragonis [17] classifies common questions types found in computer science challenge problems. Hakulinen [9] provides a survey of challenge task development. Forišek [8] gamely suggests new possible problem categories, and discusses an Internet Problem Solving Contest (IPSC) designed to escape some of the more common tropes found in ICPCand IOI-style competitions.

Despite the longevity and popularity of the traditional model for such events, it is clear that there is room for improvement. Critics point out that time-bound contest problems evaluated solely through automated black box testing discourage many of the most important pedagogical goals of modern computer science [3]. The emphasis on speed in the ICPC-style contest crowds out documentation, refinement and design. The emphasis on platform-independent programming languages and development environments, as well as black-box testing, usually discourages the use of graphics and sound, sharply limiting the creative universe of both problem tasks and their solutions.

Finally, researchers have been commenting on the lack of diversity of participants attracted by the traditional contest model since at least the 1990s [7].

\subsection{Alternative Competitions}

While the ICPC-style competition is likely to persist in some form, many alternative competition styles have been proposed to address its known shortcomings, including its lack of participant diversity [7].

Alternatives such as the Kansas City Computer Science Fair [7] and the College of Charleston's alternative competition [3] emphasize quality-of-process by judging criteria based on both technical and artistic merit, rather than just black box testing [3]. We reused these ideas in order to encourage creativity and reward artistic merit.

Others have combined CS Unplugged [14] activities with competition-style programming problems [19] to produce an effective hybrid. A wide variety of robotics-based competitions [15] allow participants to branch out beyond text-based input and output, as well as simple black-box testing.

Brebas [5] (Lithuanian for, "Beaver"), is an international, online competition series with divisions open to grades 5 through 12 , and intended for those interested in computing regardless of skill level. Individual tasks are typically short, taking 3 minutes or so to solve. Typical Brebas tasks are not programming tasks, but use a computer to solve interactive and/or multiple-choice prompts. In contrast, our work retains the focus on algorithmic design and programming, using the Scratch [12] programming language.

\subsection{Scratch Competitions}

Competitions that use Scratch or SNAP [13] take advantage of these language's attractive, block-based graphical programming environment to make the programming task more welcoming to novices. Scratch prevents standard syntax errors, and offers a simple set of primitives to manipulate sprites (graphical elements with attached method behaviors) on a stage (background). Emphasis is on animation, sound, and reactive programming features such as mouse clicks and keyboard events.

The annual Harvard SCRatch International Programming Trial (SCRIPT) ${ }^{3}$ began in 2012. Students ages 6-15 can participate in groups of $2-4$, and submit a Scratch project under one of six categories: greetings, games, music and dance, stories, simulations, and educational software. Both Georgia Tech ${ }^{4}$ and University of New Brunswick ${ }^{5}$ have also offered a Scratch-based competition in several past years similar to SCRIPT described above.

Like many other alternative competition formats, all three of these alternative Scratch competitions differ from our

\footnotetext{
${ }^{3}$ http://scratched.gse.harvard.edu/discussions/events/ scratch-competition-symposium-script-2014

${ }^{4}$ http://home.cc.gatech.edu/TeaParty/346

${ }^{5}$ http://www.unb.ca/saintjohn/sase/dept/csas/ competitions/jr-high/index.html
} 
work by focusing on online, long-term tasks, rather than an on-site track co-located with traditional ICPC-style contestants. However, all three efforts seek to accomplish similar goals to our work:

- Building students' sense of belonging in computing,

- Encouraging students to code, and

- Encouraging local teachers to teach Scratch.

The use of Scratch in the Syrian Olympiad in Informatics [10] is the clearest antecedent to our current work. Like our contest tasks, the SOI participants were given sprites and a stage, and asked to add behavior. The overall structure of the SOI differs markedly from our typical high school outreach event, and was not designed to bring a growing community of Scratch-using high school students into a parallel competition track.

\section{YEAR ONE COMPETITION}

For the Spring 2014 edition of our high school programming competition, we decided to add a new, parallel, Scratchbased track to our existing ICPC-style competition that allowed Java, Python and $\mathrm{C}++$ solutions. We knew that the Scratch Division needed to remain open-ended to promote creativity while completing in the same timespan as the other competition.

At that time, we could find no readily available resources that suggested previously successful task prompts for such a competition. Existing models (see prior section) generally relied on much longer competition times, and also on longer judging times. There were no examples of on-site, open-ended, Scratch-based tasks suitable for grades 9-12, or corresponding judging rubrics.

\subsection{Task Prompt}

The final design envisioned a single, three-hour challenge task centered on a particular, classic fairy-tale motif. Students were asked to retell the story of The Three Little Pigs.

The task description included a summary of The Three Little Pigs and key requirements such as a minimum of three scenes, repetition of appropriate dialogue, and a conclusion that emphasizes the moral of the story - that hard work shall be rewarded when challenging times come.

Participants were admonished that their work should be original. They could use any of the sprites, backgrounds, audio and other narrative elements that are available through the Scratch.mit.edu site, but should not reuse or adapt existing Scratch projects.

\subsection{Judging}

The existing PC2 contest management software was determined to be unsuitable for running the Scratch Division. Because the entries would only be judged at the end, there was no need for features handling rapid submission, testing and feedback. Moreover, the complex and somewhat intimidating default interface of the software was deemed likely to detract from the overall welcoming message behind the Scratch Division in the first place.

A panel of faculty judges evaluated work submitted using four criteria including originality, creativity, elegance, and sophistication.

The small number of Scratch Division participants in the first year allowed judging to be completed successfully. However, entirely subjective judging went slowly, and three hours per task allowed participants to produce elaborate artifacts

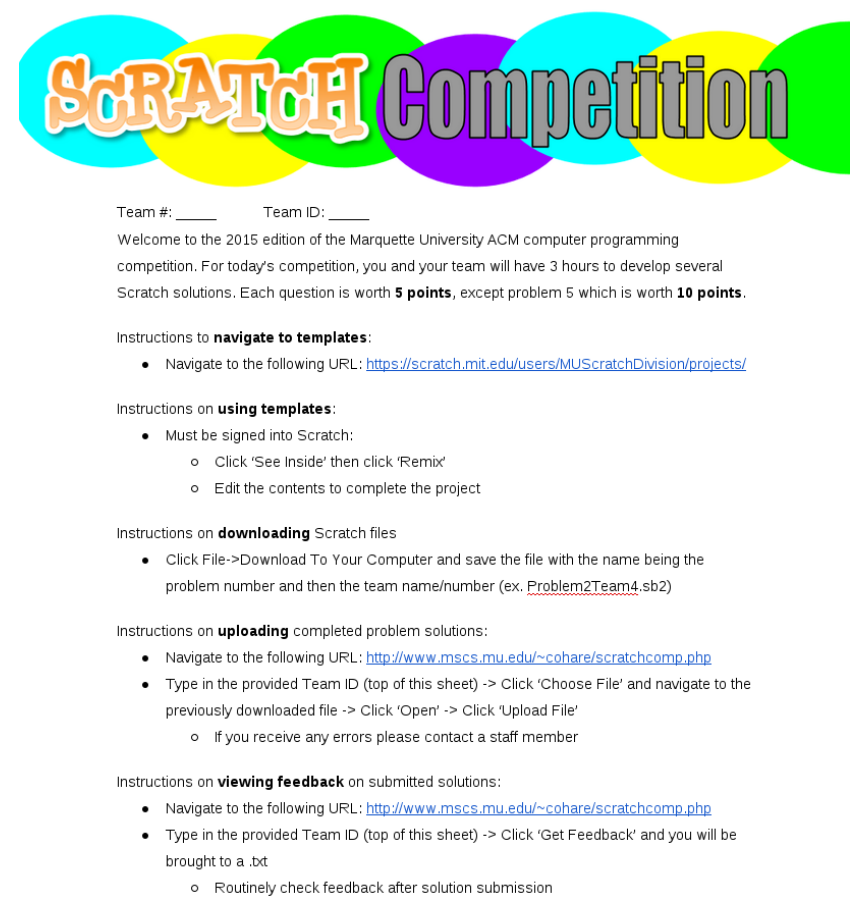

Figure 1: Scratch Division Instruction Page

that were difficult for judges to fully explore in the time allotted.

\section{YEAR TWO COMPETITION}

In the second year, we made several important changes to the structure of the Scratch Division in order to more easily scale problem judging, while retaining the distinctive character of the competition.

Competitors were still permitted to compete in pairs, or individually. While high school students were the target audience, one coach requested and received permission to bring an advanced middle school team.

The competitors were given a packet of competition questions, including detailed instructions on how to access templates and submit their work. Additionally, the packets were created to be colorful and playful (compared with the traditional, no-frills ICPC-style specifications) in order to encourage participants to maintain a creative and open mindset. (See Figure 1.)

A mix of both closed- and open-ended questions were used, meaning quicker judging for the technical points during the competition, allowing more time for the judging of creative merit.

Under the question type taxonomy suggested in [17], this task set included:

- Type 1: Developing a Solution (tasks 1, 2, 3, 4)

- Type 2: Developing a Solution that Uses a Given Module (tasks 1, 2), and

- Type 7: Completing a Given Solution (problem 1).

\subsection{Task 1 - The Maze}

For the first task, the designers sought to present the students with a straightforward, recognizable, and easy to implement question. Given a maze backdrop and a sprite, 
along with incomplete code blocks, the participants were expected to program the sprite to maneuver through the maze. They were expected to provide the test loop to determine when the sprite should stop moving (e.g. hitting a wall, crossing the finish line).

The designers intended for this problem to be solvable by all teams. Although not all teams received full marks, each team submitted a functioning copy of the sprite and maze. Not all of the teams were able to end the game successfully.

\subsection{Task 2 - The Fly Swatter}

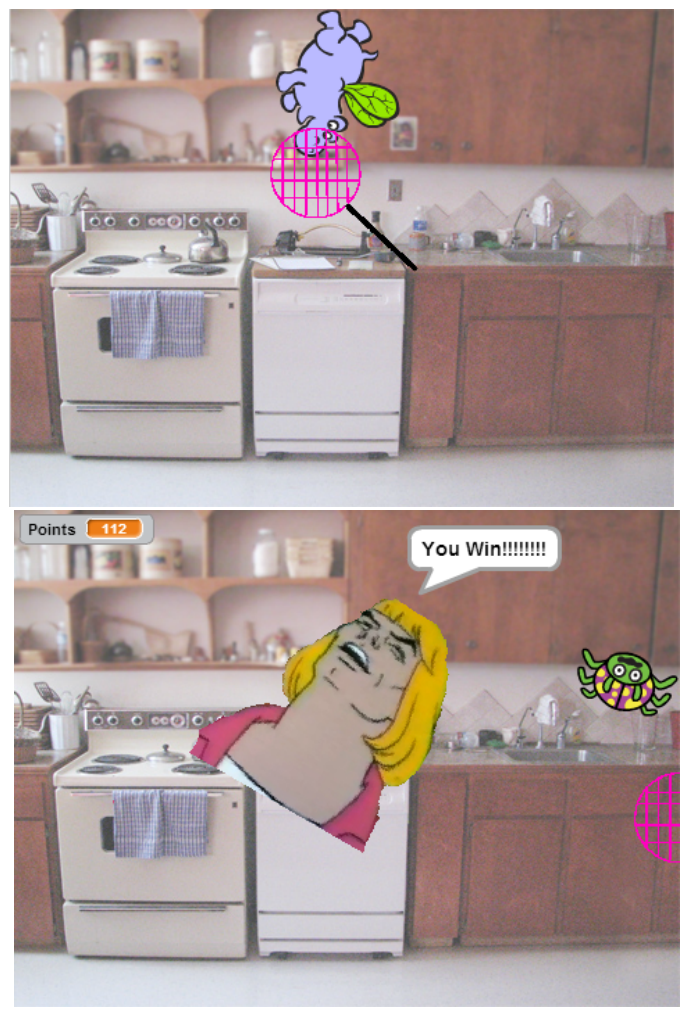

Figure 2: Flying Hippo Swatter and He-Man Sings

The second question required students to make a fly swatter game. Participants were given a fly sprite, swatter sprite, and a kitchen background. The fly was pre-programmed to randomly move around the kitchen, and the students were to map the swatter sprite to the user's mouse, "squashing" the fly sprite when clicked. The question's main objective was to see if students were able to make the game work well, which required the bug to wait a certain amount of time before moving again. If this was not present in the participant's submission, it would be difficult to win at all.

All eight of the participating groups produced a submission for this problem, with only 6 receiving full points.

Despite the relatively structured nature of this task, participants still incorporated creativity into their solutions. Some teams replaced sprites, while others created elaborate win animations, such as in Figure 2, which incorporated a winged hippopotamus instead of the simple fly, and a popular Internet meme from that month ${ }^{6}$.

\footnotetext{
${ }^{6}$ http://knowyourmeme.com/memes/he-man-sings
}

\subsection{Task 3 - Rock-Paper-Scissors}

The third task asked the participants to develop a working rock-paper-scissors game using the given sprites and their own animations. They were also required to find their own background to complement their game and animation, as well as promote the creativity of the question.

Creative solutions to the animation subtask included spinning the selection, keyboard movement for selection, and moving towards the opposing sprite.

\subsection{Task 4 - Pong}

The fourth task in the competition asked the participants to create a working two-dimensional one-player pong game (Figure 3) and for extra credit they could implement a custom artificial intelligence algorithm as a second player. This was intended to be the most challenging task in the Scratch Division.

As expected, this slightly more difficult problem did not elicit as much creative flourish as the earlier, easier problems.

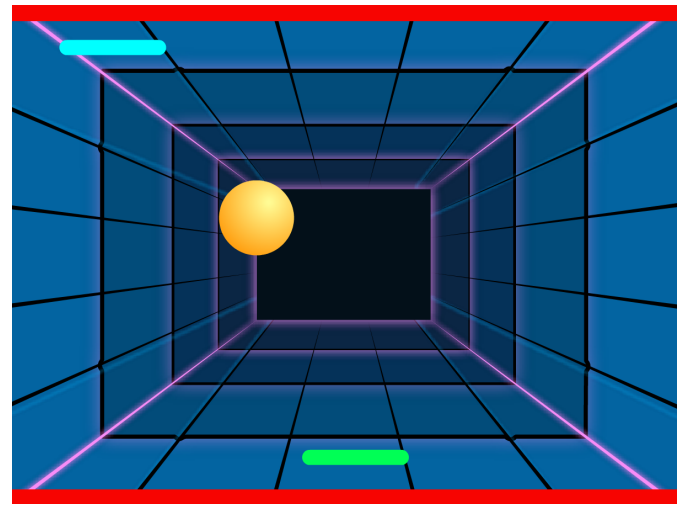

Figure 3: Task 4 - Pong

\subsection{Task 5 - Sunken Ship Narrative}

The fifth task was designed to be the most open-ended, allowing participants to demonstrate both their Scratchfluency and creativity (Figure 4). Similar to our first year competition's sole task, the fifth task provided a story prompt and directed participants to finish the story. The plot included a tale of a sunken ship; however, it was up to the participants to provide their own developments to the story, conclude it with a somewhat logical ending, and include the required elements (e.g., underwater animation, loops, at least two sound clips). The participants were also asked to include as many additional objects as they saw fit. We made sure to emphasize that creativity was the ultimate goal for this question, and informed them that it was a very important qualifier for grading.

All eight groups submitted answers to this problem, with greatly varying levels of whimsy. One group made an incredibly creative story, but had difficulty implementing every requirement of the prompt, such as the code requirements.

In an echo of results from the previous year, the most creative code made the requirements difficult for judges to identify. On the far end of the spectrum, some groups made sure to hit every program requirement for the task, but did not invest time into adding creative touches to their sub- 


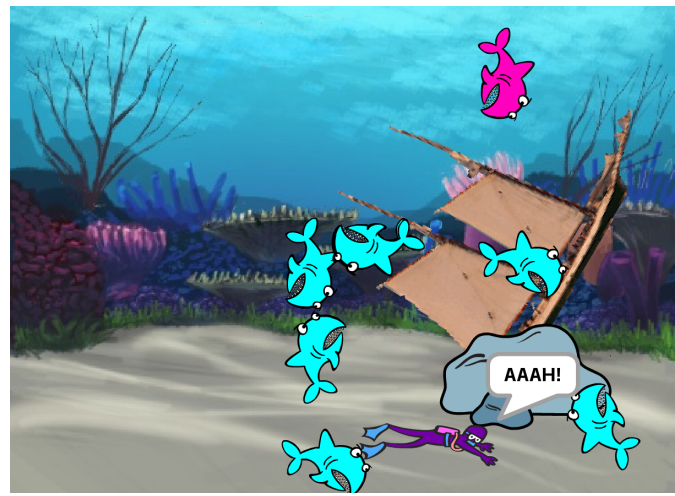

Figure 4: Task 5 given water, rock and ship sprites

mission. In the happy middle, some teams met not only the basic requirements, but retained a logical program structure.

One group developed a game in which user could maneuver a character to collect all the coins within a sunken ship while avoiding the sharks on the screen. This group even included a winning and losing screen. (See Figure 5.)

Although this question was the most difficult to grade, it provided the largest variety of solution types. This not only allowed each individual group to set themselves apart and present their personalities, but allowed us to take a look into how these groups performed "in the wild". We were thus able to observe how the participants built their own blocks of code, while allowing them to personalize it and have fun along the way.

Anecdotal feedback from the teams confirmed that this question was both the judges' and the participants' favorite.

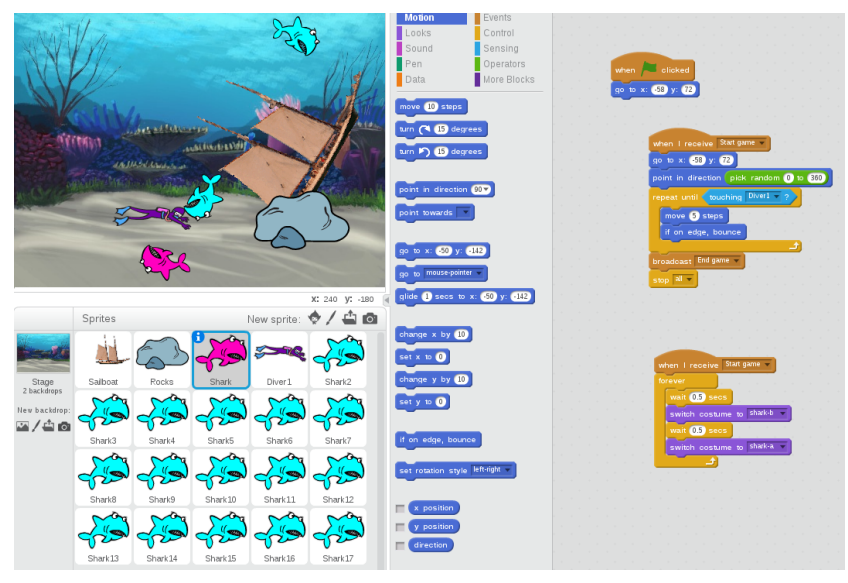

Figure 5: Task 5 Shark Sprite skin choices and Code

\subsection{Submission and Judging}

We deployed a simple, PHP-driven web submission system for the Scratch Division. This was deemed to be a simpler, lighter-weight and more theme-appropriate solution than the standard PC2 contest management software. (PC2 was still used for the Java Division.)

The judging rubric included a five point system the participants needed to fulfill in order to get full points. While grading submissions, the judges filled out corresponding feedback.txt files that marked whether their submission was cor- rect or incorrect. If incorrect, then feedback was given in order for the team to attempt corrections. The teams could view their feedback using the same aforementioned web system with unique key.

Tasks one to four were worth five points each with an extra three points of extra credit possible for task four. All partial credit was awarded three points as long as the submission had at least three of the problem's criteria working for their submission. Open-ended task five was worth ten points; seven points were awarded if they reached the minimum requirements.

\section{RESULTS AND DISCUSSION}

In total, 36 teams participated in our year two high school programming competition - 28 teams in the traditional, ICPC-style Java Division, and eight in the Scratch Division. More than 120 students from 11 high schools across our state competed from 9am-Noon, taking up all five of the computer science department's instructional laboratories, as well as many other spaces to host registration, the coaches' meeting, judging, contest headquarters, etc.

Most significantly, two of the 11 participating schools were coached by brand-new ECS teachers. Those schools had previously had no CS teacher or courses, and had sent no previous teams to our events, but this year were able to join in on the Scratch Division.

Thanks to generous support from industrial sponsors and alumni, we were able to award prizes to the top $25 \%$ of teams in both the Java and Scratch Divisions, ranging from flatpanels to Arduino starter kits, to wireless peripherals.

Feedback from coaches and participants were overwhelmingly positive. In evaluating the success of the Scratch Division, one of our most experienced ECS teachers noted that most of the tasks resembled the in-class assessments she gives her students at the end of units. We take this as confirmation that we are on the right track. The number of ECS and CSP teachers in our area is growing sharply, due in part to outreach and professional development efforts led by our institution; a fall survey of teachers in our network suggests that we can expect both divisions to grow by another $25-50 \%$ in spring of 2016.

In practice, the design of the second year competition scaled well to triple the number of Scratch Division students from the previous year.

What we did not expect was the rather creative turn the participants took with the objective-based questions. For instance, the fly swatter problem (task 2) was rather straightforward; however, a team redesigned it to track how many times the fly was clicked and when it reached one-hundredand-eleven whacks a spinning animation of a picture from the internet was displayed along with sound. The originality made it more difficult to grade some submissions, but it is the opinion of the authors that this was a worthy trade-off, and one that we look forward to making again next year.

Finally, we were surprised to learn on competition day that the contestants in the Scratch Division varied more widely in age than expected. Despite ECS being intended as a 9th-grade course, our participants from ECS schools ranged from middle school students to high school seniors.

The total number of Scratch Division participants was still less than 20 this year, so it is not yet feasible to make statistically valid claims about the demographic shift we are seeing in the population participating in the Scratch Divi- 
sion. Anecdotally, the Scratch Division has had a better gender balance than the Java Division in both years.

\subsection{Future Work}

The originality and creativity that some students implemented indicates a need for more open ended questions. For several prompts, we outlined many of the steps and provided a template of sprites and some code fragments that contestants should use. This may have restricted some of the creativity that students could display. In the future, it may serve us better to give a general paragraph of what we require, and expect groups to take different routes to completing the prompt.

Moving forward, we expect to grow the competition next year, as more schools are affected by efforts to increase the number of new CS teachers in the state. We are considering creating a third division, based on previous work on CS Fairs, that would directly model one of the nonprogramming performance tasks expected for AP CSP.

As the number of participants grows, we hope to complete a statistically valid analysis of the differences between the populations in the two tracks. Our hypothesis is that the Scratch Division attracts a higher percentage of women than the current Java Division, and draws teams from schools with a larger population of underrepresented students.

\section{CONCLUSION}

We have presented the structure and rationale for an alternative, Scratch-based track that we have added to our existing ICPC-style high school programming competition. The design of this track incorporates both relatively constrained tasks that can be quickly judged, and more open-ended tasks that can be rewarded for creativity and artistic merit. Our overriding goal was to include in our annual outreach event the growing number of Scratch programmers coming out of broader introductory CS courses such as ECS and AP CSP. Students participate in the same, on-campus event, with a different set of tasks, proctors and judges, and participate in the same awards ceremony.

The competition ran well, and attracted markedly more participants than the previous years. The presence of a Scratch Division allowed new schools to participate who had previously never sent a team to such an event. Furthermore, while numbers are still small, it seems likely that the demographic balance of the Scratch Division will at least initially beat the traditional Java Division competition.

\section{Acknowledgments}

The authors would like to thank the high school teachers of the CSTA Wisconsin-Dairyland chapter for their guidance and support in creating our event, and for bringing their teams of students. We are grateful to the Marquette University student chapters of ACM and Upsilon Pi Epsilon for the logistical and volunteer support that made our events possible. Aspects of the competition were supported by generous donations from Marquette computer science alumni as well as local employers, such as Rockwell Automation and Direct Supply.

Some of the authors were supported in part by NSF CE21 grant \#CNS-1339392, the results of which are driving the need for high school programming contests that appeal to a broader audience in our region.

\section{REFERENCES}

[1] American Computer Science League. http://www.acsl.org/, Accessed: 2015-05-14.

[2] Association for Computing Machinery (ACM). ICPC factsheet. http://icpc.baylor.edu/worldfinals/pdf/Factsheet.pdf, Accessed: 2015-05-14.

[3] J. F. Bowring. A new paradigm for programming competitions. In Proceedings of the 39th SIGCSE Technical Symposium on Computer Science Education, SIGCSE '08, pages 87-91, Portland, OR, 2008. ACM.

[4] College Board. Computer science: Principles. http://www.csprinciples.org/, Accessed: 2015-05-14.

[5] V. Dagiene and G. Futschek. Bebras, a contest to motivate students to study computer science and develop computational thinking. In WCCE 2013: Tenth World Conference on Computers in Education, Torun, Poland, July 2013.

[6] Exploring Computer Science. Curriculum v6.0. http://www.exploringcs.org/, Accessed: 2015-05-14.

[7] S. Fitzgerald and M. L. Hines. The computer science fair: An alternative to the computer programming contest. In Proceedings of the Twenty-seventh SIGCSE Technical Symposium on Computer Science Education, SIGCSE '96, pages 368-372, Philadelphia, PA, 1996. ACM.

[8] M. Forišek. Pushing the boundary of programming contests. Olympiads in Informatics, 7:23-35, 2013. ISSN 1822-7732.

[9] L. Hakulinen. Survey on informatics competitions: Developing tasks. Olympiads in Informatics, 5:12-25, 2011. ISSN 1822-7732.

[10] A. Idlbi. Taking kids into programming (contests) with Scratch. Olympiads in Informatics, 3:17-25, 2009. ISSN 1822-7732.

[11] International Olympiad in Informatics. http://www.ioinformatics.org/, Accessed: 2015-05-14.

[12] MIT Media Lab. Scratch. https://scratch.mit.edu/, Accessed: 2015-05-14.

[13] U. of California at Berkeley. Snap (build your own blocks). https://snap.berkeley.edu/, Accessed: 2015-05-14.

[14] U. of Canterbury. CS unplugged: Computer science without a computer. http://csunplugged.org/, Accessed: 2015-05-14.

[15] P. Petrovič. Ten years of creative robotics contests. In Proceedings of Select Papers, ISSEP 2011: 5th International Conference on Informatics in Schools: Situation, Evolution and Perspectives, pages 201-212, Bratislava, Slovakia, October 2011.

[16] W. Pohl. Computer science contests for secondary school students: Approaches to classification. Informatics in Education, 5(1):125-132, 2006.

[17] N. Ragonis. Type of questions - the case of computer science. Olympiads in Informatics, 6:115-132, 2012. ISSN 1822-7732.

[18] USA Computing Olympiad. http://www.usaco.org/, Accessed: 2015-05-14.

[19] J. Voigt, T. Bell, and B. Aspvall. A New Learning Paradigm: Competition Supported by Technology, pages 207-234. CEDETEL, Boecillo, Spain, 2009. 